The Journal of Fourier Analysis and Applications

\title{
Wavelets on the Two-Sphere and other Conic Sections
}

\author{
Jean-Pierre Antoine and Pierre Vandergheynst
}

Communicated by ???

\begin{abstract}
We survey the construction of the continuous wavelet transform (CWT) on the two-sphere. Then we discuss the discretization of the spherical CWT, obtaining various types of discrete frames. Finally we give some indications on the construction of a CWT on other conic sections.
\end{abstract}

\section{Introduction}

Many situations in physics, astronomy and medicine yield data on spherical manifolds. Also, some data may live on a two-sheeted hyperboloid, in cosmology for instance (an open expanding model of the universe). In optics also, in the catadioptric image processing, where a sensor overlooks a mirror with the shape of a hyperboloid or a paraboloid. So one needs a suitable analysis tool for data living on a non-Euclidean manifold. This suggests to adapt the continuous wavelet transform (CWT) to the three manifolds above. In addition, all three constitute the so-called conic sections, generated by intersecting a double cone by a plane. Thus one may also try to design a unified $C W T$ formalism for all conic sections at once.

In this paper, we shall review the construction of the continuous wavelet transform (CWT) on the two-sphere, both by the group-theoretical approach and the geometrical method based on conformal invariance. Next, we briefly discuss the discretization of this spherical CWT, leading to various types of frames, first half-continuous ones (only the scale is discretized), then fully discrete ones. Finally we give some brief indications on the construction of a CWT on the other conic sections.

Math Subject Classifications. 42C15, 42C40, 65T60 .

Keywords and Phrases. wavelets, continuous wavelet transform, frames, conic sections. 


\section{The CWT on the two-sphere}

Let us begin with the two-sphere $S^{2}$. Fourier analysis on $S^{2}$ is standard, but cumbersome, since it amounts to work with expansions in spherical harmonics. While the latter constitute an orthonormal basis of $L^{2}\left(S^{2}\right)$, they are not localized at all on the sphere, so that Fourier analysis is global. Actually, there are specific combinations of spherical harmonics which are well localized, the so-called spherical harmonics kernels [27], but then one loses the simplicity of an orthonormal basis.

Thus it is not surprising that alternative solutions have been proposed by several authors. We may quote, for instance, Gabor analysis on the tangent bundle [34]; frequential wavelets, based on spherical harmonics [16]; or diffusion methods with a heat equation [10]. Discrete wavelets on the sphere have also been designed, using an $S^{2}$ multiresolution analysis. For instance, Haar wavelets on a triangulation of $S^{2}$ and refined with the lifting scheme [32]; $C^{1}$ wavelets constructed by a factorization of the refinement matrices [35]; or wavelets obtained by radial projection from a polyhedron incribed in the sphere (typically locally supported spline wavelets on spherical triangulations) $[29,30]$. References to the (vast) literature on discrete spherical wavelets may be found in $[29,35]$ for earlier work and in [21] for recent work.

However, various problems plague those constructions, such as an inadequate notion of dilation, the lack of wavelet localization, the excessive rigidity of the wavelets obtained, the lack of directionality, etc. In this respect, the continuous wavelet transform (CWT) has many advantages: locality is controlled by dilation, the wavelets are easily transported around the sphere by rotations from $\mathrm{SO}(3)$, efficient algorithms are available. Holschneider [19] was the first to build a genuine spherical CWT, but his construction involves several assumptions and lacks a geometrical feeling. In particular, it contains a parameter that has to be interpreted as a dilation parameter, but whose geometrical meaning is unclear. A satisfactory solution was obtained in a series of papers from our groups $[1,2,3,9]$ that yield a rigorous and efficient spherical CWT. A further simplification was obtained later by invoking conformal arguments [36]. Of course, in practice, the usual two-dimensional CWT in the plane is discretized and replaced with suitable discrete frames. Thus, to complete the picture, one needs to design discrete spherical wavelet frames as well, and this was indeed realized in [9].

This section is devoted to a rapid survey of the works mentioned above. For 2-D wavelets in general, we refer to our recent monograph [4]. For the convenience of the reader, we have collected in an appendix some basic notions of group theory that are used in the text. 


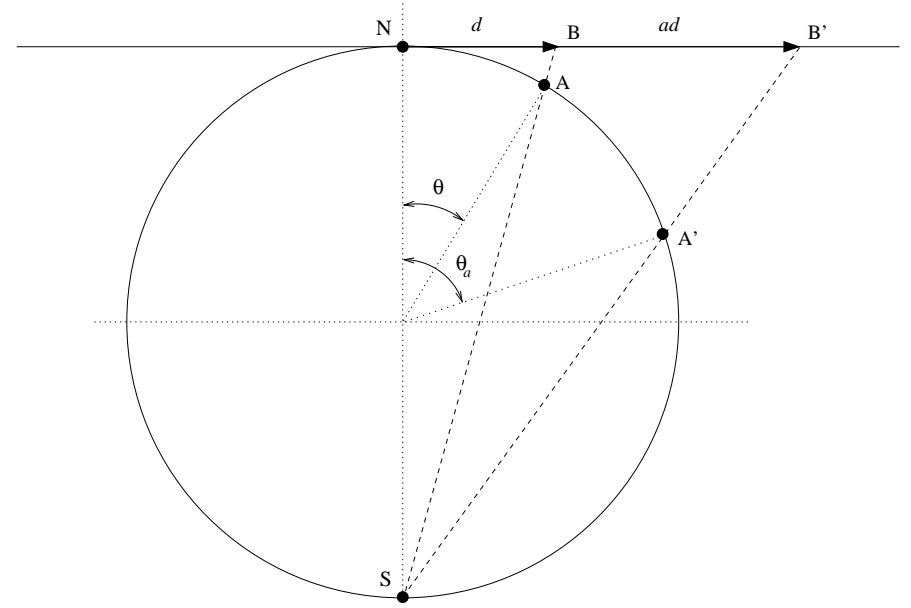

FIGURE 1 Visual meaning of the stereographic dilation on $S^{2}$.

\subsection{The spherical CWT: heuristics}

As we have learned from the previous cases, the design of a CWT on a given manifold $X$ starts by identifying the operations one wants to perform on the finite energy signals living on $X$, that is, functions in $L^{2}(X, d \nu)$, where $\nu$ is a suitable measure on $X$. Next one realizes these operations by unitary operators on $L^{2}(X, d \nu)$ and one looks for a possible group-theoretical derivation.

In the case of the two-sphere $S^{2}$, the required transformations are of two types: (i) motions, which are realized by rotations $\varrho \in \mathrm{SO}(3)$, and (ii) dilations of some sort by a scale factor $a \in \mathbb{R}_{+}^{*}$. The problem is how to define properly the dilation on the sphere $S^{2}$.

A possible solution is to use a (radial) stereographic dilation on $S^{2}$, which is obtained in three steps (Figure 1): (i) given a point $\mathrm{A} \in S^{2}$, different from the South Pole $S$, project it stereographically to the point $B$ in the plane tangent to the sphere at the North Pole N; (ii) dilate $B$ radially in the usual way to $B^{\prime}$; and (iii) project back B' to the sphere, which yields $A^{\prime}$. The map $A \mapsto A^{\prime}$ is the required spherical dilation around $N$. In order to dilate around any other point $\mathrm{C}$, just bring it to $\mathrm{N}$ by a rotation $\varrho \in \mathrm{SO}(3)$, dilate as above, and go back to $C$ by the inverse rotation $\varrho^{-1}$.

Our Hilbert space is $L^{2}\left(S^{2}, d \mu\right)$, where $d \mu=\sin \theta d \theta d \varphi, \theta \in[0, \pi]$ is the latitude angle, $\varphi \in[0,2 \pi)$ the longitude angle, $\omega=(\theta, \varphi) \in S^{2}$, and we use the orthonormal basis of spherical harmonics $\left\{Y_{l}^{m}, l \in \mathbb{N},|m| \leqslant l\right\}$.

Then the operations just defined have a natural realization by unitary operators in $L^{2}\left(S^{2}, d \mu\right)$ :

$$
\begin{aligned}
& \text {. rotation } R_{\varrho}:\left(R_{\varrho} f\right)(\omega)=f\left(\varrho^{-1} \omega\right), \varrho \in \mathrm{SO}(3), \\
& \text {. dilation } D_{a}:\left(D_{a} f\right)(\omega)=\lambda(a, \theta)^{1 / 2} f\left(\omega_{1 / a}\right), a \in \mathbb{R}_{+}^{*} \text {. }
\end{aligned}
$$


In these relations, $\omega_{a}=\left(\theta_{a}, \varphi\right), \theta_{a}$ is defined by $\tan \frac{\theta_{a}}{2}=a \tan \frac{\theta}{2}$ for $a>0$ and the normalization factor $\lambda(a, \theta)^{1 / 2}$ (variously called cocycle or RadonNikodym derivative) is needed for compensating the noninvariance of the measure $\mu$ under dilation. Explicitly, this factor is given by

$$
\lambda(a, \theta)=\frac{4 a^{2}}{\left[\left(a^{2}-1\right) \cos \theta+\left(a^{2}+1\right)\right]^{2}} .
$$

Note that the rotation $\varrho$ may be factorized into 3 rotations (Euler angles): $R_{\varrho}=R_{\varphi}^{\mathrm{z}} R_{\theta}^{\mathrm{y}} R_{\chi}^{\mathrm{z}}, \varphi, \chi \in[0,2 \pi), \theta \in[0, \pi]$.

The question now is, can one derive a CWT from these ingredients, as for the 2-D plane CWT? Is this transformation unique?

\subsection{The group-theoretical method}

According to the general scheme [4], a possible way of answering the question is to use the general coherent state formalism relying on square integrable representations of a suitable transformation group. Thus we start from the affine transformations on $S^{2}$, namely, motions (here, rotations) and dilations. But a problem arises immediately. On the one hand, motions $\varrho \in \mathrm{SO}(3)$ and dilations by $a \in \mathbb{R}_{*}^{+}$do not commute. On the other hand, it is impossible to build a semidirect product of $\mathrm{SO}(3)$ and $\mathbb{R}_{*}^{+}$, since $\mathrm{SO}(3)$ does not have any outer automorphism, so that the only extension of $\mathrm{SO}(3)$ by $\mathbb{R}_{*}^{+}$is their direct product. However, this contradiction may be evaded if one embeds the two factors into the Lorentz group $\mathrm{SO}_{o}(3,1)$, by the Iwasawa decomposition $[8,18]$ :

$$
\mathrm{SO}_{o}(3,1)=\mathrm{SO}(3) \cdot A \cdot N \text {, }
$$

where $\mathrm{SO}(3)$ is the maximal compact subgroup of $\mathrm{SO}_{o}(3,1), A \simeq \mathrm{SO}_{o}(1,1) \simeq$ $\mathbb{R} \simeq \mathbb{R}_{*}^{+}$(boosts in the $z$-direction) and $N \simeq \mathbb{C}$. This procedure is justified by the fact that the Lorentz group $\mathrm{SO}_{o}(3,1)$ is the conformal group both of the sphere $S^{2}$ and of the tangent plane $\mathbb{R}^{2}$.

Next we have to compute the action of the Lorentz group on the sphere. The stability subgroup of the North Pole is $P=\mathrm{SO}_{z}(2) \cdot A \cdot N$. Thus $\mathrm{SO}_{o}(3,1) / P \simeq \mathrm{SO}(3) / \mathrm{SO}(2) \simeq S^{2}$, so that $\mathrm{SO}_{o}(3,1)$ acts transitively on $S^{2}$. Then an explicit computation with help of the Iwasawa decomposition (2.4) shows that the pure dilation by $a$, realized as a Lorentz boost along the $z$-axis, coincides with the stereographic dilation (2.2).

Going over to the Hilbert space, we find [33] that the Lorentz group $\mathrm{SO}_{o}(3,1)$ has a unitary irreducible representation (UIR) in $L^{2}\left(S^{2}, d \mu\right)$ :

$$
[U(g) f](\omega)=\lambda(g, \omega)^{1 / 2} f\left(g^{-1} \omega\right), \text { for } g \in \operatorname{SO}_{o}(3,1), f \in L^{2}\left(S^{2}, d \mu\right),
$$

where $\lambda(g, \omega) \equiv \lambda(a, \theta)$ is the Radon-Nikodym derivative (2.3).

Thus the parameter space of spherical wavelets is the homogeneous space $X=\mathrm{SO}_{o}(3,1) / N \simeq \mathrm{SO}(3) \cdot \mathbb{R}_{*}^{+}$, which is not a subgroup of $\mathrm{SO}_{o}(3,1)$. 
Therefore, in order to apply the general formalism, we must introduce a section $\sigma: X \rightarrow \mathrm{SO}_{o}(3,1)$ and consider the reduced representation $U(\sigma(\varrho, a))$. Choosing the natural (Iwasawa) section $\sigma(\varrho, a)=\varrho a, \varrho \in \mathrm{SO}(3), a \in A$, we obtain

$$
U(\sigma(\varrho, a))=U(\varrho a)=U(\varrho) U(a)=R_{\varrho} D_{a},
$$

exactly as before, in (2.1)-(2.2).

The following three propositions show that the representation (2.6) has all the properties that are required to generate a useful CWT. First of all, it is square integrable on the quotient manifold $X=\mathrm{SO}_{o}(3,1) / N \simeq \mathrm{SO}(3) \cdot \mathbb{R}_{*}^{+}$. For simplicity, we shall identify these two isomorphic manifolds.

Proposition 2.1 The UIR (2.5) is square integrable on $X$, that is, there exist nonzero (admissible) vectors $\psi \in L^{2}\left(S^{2}, d \mu\right)$ such that

$$
\int_{0}^{\infty} \frac{d a}{a^{3}} \int_{\mathrm{SO}(3)} d \varrho|\langle U(\sigma(\varrho, a)) \psi \mid \phi\rangle|^{2}:=\left\langle\phi \mid A_{\psi} \phi\right\rangle<\infty, \text { for all } \phi \in L^{2}\left(S^{2}, d \mu\right) .
$$

Here de is the left invariant (Haar) measure on $S O(3)$.

The resolution operator (also called frame operator) $A_{\psi}$ is diagonal in Fourier space (i.e., it is a Fourier multiplier):

$$
\widehat{A_{\psi} f}(l, m)=G_{\psi}(l) \widehat{f}(l, m),
$$

where

$$
G_{\psi}(l)=\frac{8 \pi^{2}}{2 l+1} \sum_{|m| \leqslant l} \int_{0}^{\infty} \frac{d a}{a^{3}}\left|\widehat{\psi}_{a}(l, m)\right|^{2}, \quad \text { for all } l \in \mathbb{N},
$$

and $\widehat{\psi}_{a}(l, m)=\left\langle Y_{l}^{m} \mid \psi_{a}\right\rangle$ is a Fourier coefficient of $\psi_{a}=D_{a} \psi$.

Next, we have an exact admissibility condition on the wavelets (this condition was also derived by Holschneider [19] in a somewhat ad hoc way).

Proposition 2.2 An admissible wavelet is a function $\psi \in L^{2}\left(S^{2}, d \mu\right)$ for which there exists a positive constant $c<\infty$ such that

$$
G_{\psi}(l) \leqslant c, \text { for all } l \in \mathbb{N} .
$$

Equivalently, the function $\psi \in L^{2}\left(S^{2}, d \mu\right)$ is an admissible wavelet if and only if the resolution operator $A_{\psi}$ is bounded and invertible.

As in the plane case [4], there is also a weaker admissibility condition on $\psi$ :

$$
\int_{S^{2}} d \mu(\omega) \frac{\psi(\theta, \varphi)}{1+\cos \theta}=0 .
$$


Here too, this condition is only necessary in general, but it becomes sufficient under mild regularity conditions on $\psi$. This is clearly similar to the "zero mean" condition of wavelets on the line or the plane. As in the flat case, it implies that the spherical CWT acts as a local filter, in the sense that it selects the components of a signal that are similar to $\psi$, which is assumed to be well localized.

Finally, our spherical wavelets generate continuous frames. Indeed:

Proposition 2.3 For any admissible wavelet $\psi$ such that $\int_{0}^{2 \pi} d \varphi \psi(\theta, \varphi) \not \equiv$ 0 , the family $\left\{\psi_{a, \varrho}:=R_{\varrho} D_{a} \psi: a>0, \varrho \in \mathrm{SO}(3)\right\}$ is a continuous frame, that is, there exist two constants $\mathrm{m}>0$ and $\mathrm{M}<\infty$ such that

$$
\mathrm{m}\|\phi\|^{2} \leqslant \int_{0}^{\infty} \frac{d a}{a^{3}} \int_{\mathrm{SO}(3)} d \varrho\left|\left\langle\psi_{a, \varrho} \mid \phi\right\rangle\right|^{2} \leqslant \mathrm{M}\|\phi\|^{2}, \text { for all } \phi \in L^{2}\left(S^{2}, d \mu\right),
$$

or, equivalently, there exist two positive constants $d>0$ and $c<\infty$ such that

$$
d \leqslant G_{\psi}(l) \leqslant c, \text { for all } l \in \mathbb{N}
$$

(in other words, the operators $A_{\psi}$ and $A_{\psi}^{-1}$ are both bounded).

Note that the condition $\int_{0}^{2 \pi} d \varphi \psi(\theta, \varphi) \not \equiv 0$ is automatically satisfied for any nonzero axisymmetric (zonal) wavelet. Also the frame so obtained is most probably not tight (we don't have a definite result).

A simple example of admissible spherical wavelet is the Difference of Gaussians spherical wavelet (SDOG), obtained by lifting onto the sphere the usual plane DOG wavelet (Figure 2). More precisely, for $\phi(\theta, \varphi)=$ $\exp \left(-\tan ^{2}\left(\frac{\theta}{2}\right)\right)$, the SDOG wavelet is defined as

$$
\psi_{G}^{(\alpha)}(\theta, \varphi)=\phi(\theta, \varphi)-\frac{1}{\alpha}\left[D_{\alpha} \phi\right](\theta, \varphi), \quad \text { for } \alpha>0 .
$$

Using the previous results, we may now introduce the spherical CWT.

Definition 2.4 Given the admissible wavelet $\psi$, the spherical CWT of a function $f \in L^{2}\left(S^{2}, d \mu\right)$ with respect to $\psi$ is defined as

$$
W_{f}(\varrho, a):=\left\langle\psi_{a, \varrho} \mid f\right\rangle=\int_{S^{2}} d \mu(\omega) \overline{\left[R_{\varrho} D_{a} \psi\right](\omega)} f(\omega)=\left(\psi_{a} \star f\right)(\varrho) .
$$

In the last equality, $\star$ denotes a spherical correlation.

According to the general coherent state formalism, there is a reconstruction formula. For any $f \in L^{2}\left(S^{2}, d \mu\right)$ and $\psi$ an admissible wavelet such that $\int_{0}^{2 \pi} d \varphi \psi(\theta, \varphi) \not \equiv 0$, one has

$$
f(\omega)=\int_{0}^{\infty} \frac{d a}{a^{3}} \int_{\mathrm{SO}(3)} d \varrho W_{f}(\varrho, a)\left[A_{\psi}^{-1} R_{\varrho} D_{a} \psi\right](\omega) .
$$




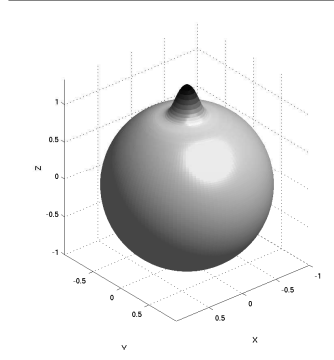

(a)

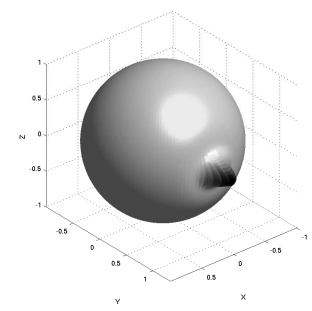

(b)

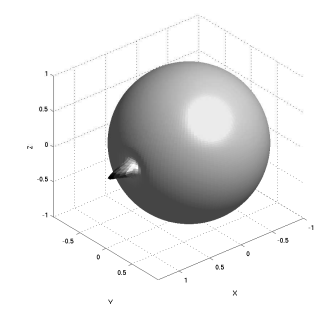

(c)

FIGURE 2 The spherical wavelet $\psi_{G}^{(\alpha)}$ wavelet, for $\alpha=1.25$ : (a) Original $(a=0.125)$; (b) Rotated; (c) Rotated and scaled $(a=0.0625)$.

Correspondingly, instead of the familiar isometry property, one gets a Plancherel relation:

$$
\|f\|^{2}=\int_{0}^{\infty} \frac{d a}{a^{3}} \int_{\mathrm{SO}(3)} d \varrho W_{f}(\varrho, a) \overline{\widetilde{W}_{f}(\varrho, a)}
$$

where

$$
\widetilde{W}_{f}(\varrho, a):=\left\langle\widetilde{\psi}_{a, \varrho} \mid f\right\rangle=\left\langle A_{\psi}^{-1} R_{\varrho} D_{a} \psi \mid f\right\rangle .
$$

The new fact here is the occurrence of the inverse resolution operator $A_{\psi}^{-1}$ in these formulas. This results from the square integrability of the representation (2.5) over the quotient space $X$, instead of the group itself.

Note that all the formulas simplify if the wavelet is axisymmetric. In particular, the third Euler angle $\chi$ drops out in $R_{\varrho}$, so that motions are now indexed by points $\omega \in S^{2}$. Hence we may write $R_{[\omega]}$ instead of $R_{\varrho}$. The corresponding wavelet family is thus $\left\{\psi_{a, \omega}:=R_{[\omega]} D_{a} \psi: a>0, \omega \in S^{2}\right\}$, and it is a frame under the same condition as in Proposition 2.3. Otherwise there is no essential modification.

In order to illustrate the capabilities of our spherical CWT, we present first, in Figure 3, an academic example, namely, the transform of the characteristic function of a triangle with apex at the North Pole, $0^{\circ} \leqslant \theta \leqslant$ $50^{\circ}, 0^{\circ} \leqslant \varphi \leqslant 90^{\circ}$, obtained with the spherical DOG wavelet $\psi_{G}^{(\alpha)}$ given in (2.13), for $\alpha=1.25$. The transform is shown at three different, gradually smaller, scales, $a=0.2,0.1$ and 0.035. As expected, it vanishes inside the triangle, and presents a "wall" along the contour, with sharp peaks at each vertex, and the North Pole does not play any particular role. This example confirms that the spherical CWT behaves exactly as its plane counterpart.

Next we present, in Figure 4, a real life example, namely, the analysis of an image of the Milky Way, based on data from the Hipparcos and Tycho Star Catalogues. 


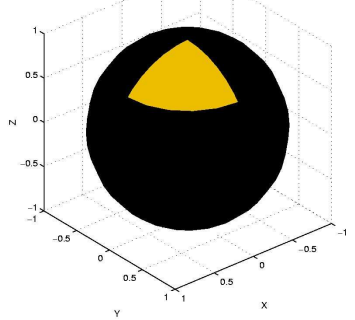

(a)

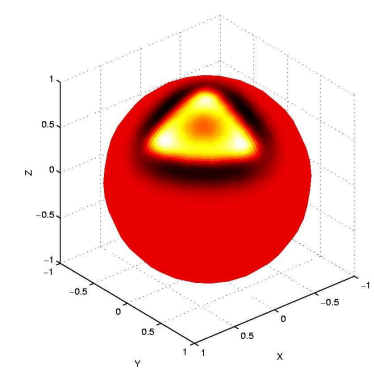

(c)

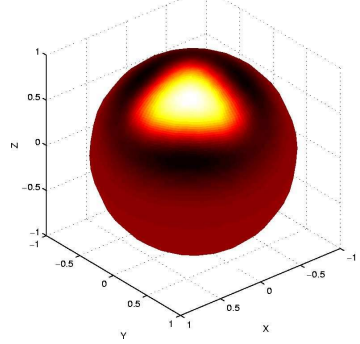

(b)

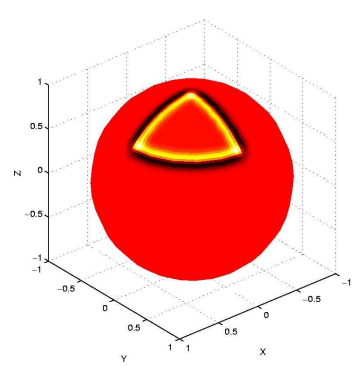

(d)

FIGURE 3 Spherical wavelet transform of the characteristic function of a triangle, obtained with the spherical DOG wavelet $\psi_{G}^{(\alpha)}$, for $\alpha=1.25$. (a) Original image. The transform is shown at three gradually smaller scales, (b) $a=0.2$; (c) $a=0.1$; and (d) $a=0.035$.

\subsection{The Euclidean limit}

The geometry of the sphere suggests that, when the radius $R$ increases to infinity, the CWT on $S^{2}$ should tend locally to the CWT on the tangent plane at the North Pole. This condition, imposed for consistency reasons by Holschneider [19], may actually be derived in the group-theoretical approach, using the technique of group contraction, with the sphere radius as parameter, $R \rightarrow \infty$.

The limit $R \rightarrow \infty$ must be taken at several successive stages. The result of the analysis is the following.

(1) For the groups

$$
\mathrm{SO}_{o}(3,1)=\mathrm{SO}(3) \cdot \mathbb{R}_{*}^{+} \cdot N \quad \longrightarrow \quad \mathbb{R}^{2} \rtimes \mathrm{SIM}(2)
$$

Thus the parameter space $\mathrm{SO}(3) \cdot \mathbb{R}_{*}^{+}$, which is not a group, becomes in the limit the group $\operatorname{SIM}(2)$, that is, precisely the group underlying the 2-D plane CWT. 


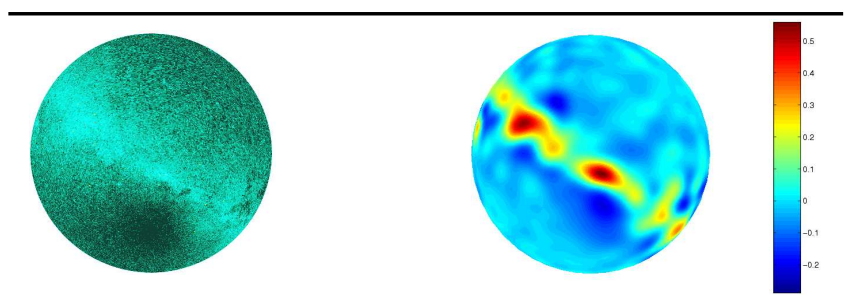

(a)

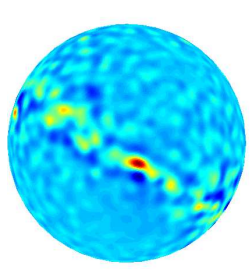

(c)

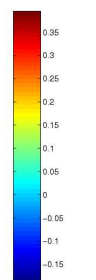

0.15

(c)

FIGURE 4 Spherical wavelet transform of an image of the Milky Way. (a) Original image. The transform is shown at three successive scales, (b) $a=0.08$; (c) $a=0.04$; (d) $a=0.02$.

(2) For the group actions

Let us replace the sphere $S^{2}$ by the sphere $S_{R}^{2}$ of radius $R$. Then:

$$
\text { action of } \sigma(X) \subset \mathrm{SO}_{o}(3,1) \text { on } S_{R}^{2} \longrightarrow \text { action of } \operatorname{SIM}(2) \text { on } \mathbb{R}^{2} \text {. }
$$

(3) For the representations

Define a family of representations $U_{R}$ on $L^{2}\left(S_{R}^{2}, d \mu_{R}(\omega)\right)$, where $d \mu_{R}(\omega)=$ $R^{2} d \mu(\omega)$, by

$$
U_{R}(\varrho, a):=U\left(\sigma\left(\varrho, a R^{-1}\right)\right)
$$

where $U$ is the representation of $\operatorname{SO}_{o}(1,3)$ given in (2.5) and (2.6). Then $U_{R} \rightarrow U$ as $R \rightarrow \infty$, as a strong limit on a dense set $[11,12]$.

(4) For the CWT on $S^{2}$

Let $\psi(\vec{x}) \in L^{2}\left(\mathbb{R}^{2}, d^{2} \vec{x}\right)$ and $\psi_{R}=\Pi_{R}^{-1} \psi$, where $\Pi_{R}: L^{2}\left(S_{R}^{2}, d \mu_{R}(\omega)\right) \rightarrow$ $L^{2}\left(\mathbb{R}^{2}, d^{2} \vec{x}\right)$ is the unitary map induced by the stereographic projection (see (2.18) below). Then

$$
G_{\psi_{R}}(l) \leqslant c(\text { for all } l \in \mathbb{N}) \quad \stackrel{R \rightarrow \infty}{\longrightarrow} \quad c_{\psi} \sim \int_{\mathbb{R}^{2}}|\widehat{\psi}(\vec{k})|^{2} \frac{d^{2} \vec{k}}{|\vec{k}|^{2}}<\infty .
$$

Thus admissible vectors on $S^{2}$ correspond to admissible vectors on $\mathbb{R}^{2}$, i.e., the Euclidean limit holds. In summary, for $\psi=\lim _{R \rightarrow \infty} \Pi_{R} \psi_{R}$ : 


$$
\begin{aligned}
& \psi_{R} \text { admissible on } S_{R}^{2} \Longrightarrow \int_{S_{R}^{2}} d \mu_{R}(\omega) \frac{\psi_{R}(\omega)}{1+\cos \theta}=0 \\
& \Downarrow \\
& \psi \text { admissible on } \mathbb{R}^{2} \quad \Longrightarrow \quad \int_{\mathbb{R}^{2}} d^{2} \vec{x} \psi(\vec{x})=0 .
\end{aligned}
$$

To give an example, take the Difference of Gaussians wavelet. When $R \rightarrow \infty$, the SDOG wavelet on $S_{R}^{2}$ tends to the usual DOG wavelet on $\mathbb{R}^{2}$.

\subsection{The geometrical or conformal method}

The group-theoretical method discussed so far yields an asymptotic connection with the plane CWT, via the Euclidean limit $R \rightarrow \infty$. In fact, there is also a direct connection (unitary map) through the inverse stereographic projection and it is uniquely specified by geometrical considerations, as we show now. The result is that one obtains uniquely the spherical CWT from the plane (Euclidean) one, simply by lifting everything from the tangent plane to the sphere by inverse stereographic projection, the wavelets, the admissibility conditions, the directionality or steerability properties [36].

(1) Uniqueness of the stereographic projection

Let $\mathrm{p}: S^{2} \backslash\{\mathrm{S}\} \rightarrow \mathbb{R}^{2}$ be a radial diffeomorphism from the 2 -sphere to the tangent plane at the North Pole:

$$
\mathrm{p}(\theta, \varphi)=(r(\theta), \varphi) \quad \text { with inverse } \quad \mathrm{p}^{-1}(r, \varphi)=(\theta(r), \varphi) .
$$

Assume in addition that $\mathrm{p}$ is a conformal map, i.e., it preserves angles. Then $r(\theta)=2 \tan \frac{\theta}{2}$, i.e., $\mathrm{p}$ is the stereographic projection.

(2) Uniqueness of the stereographic dilation

Let $D_{a}$ be a radial dilation on the sphere $S^{2}$ :

$$
D_{a}(\theta, \varphi)=\left(\theta_{a}(\theta), \varphi\right) .
$$

Assume $D_{a}$ is a conformal diffeomorphism. Then one has uniquely:

$$
\tan \frac{\theta_{a}}{2}=a \tan \frac{\theta}{2}
$$

i.e., $D_{a}$ is the stereographic dilation (2.2).

Thus one obtains an equivalence between the two wavelet formalisms. Let $\Pi: L^{2}\left(S^{2}, d \mu\right) \rightarrow L^{2}\left(\mathbb{R}^{2}, d^{2} \vec{x}\right)$ be the unitary map induced by the stereographic projection (note that $\Pi=\Pi_{R}$ for $R=1$, as given in Section 2.3):

$$
[\Pi F](\vec{x})=\frac{1}{1+(r / 2)^{2}} F\left(\mathrm{p}^{-1}(\vec{x})\right), \quad F \in L^{2}\left(S^{2}, d \mu\right),
$$




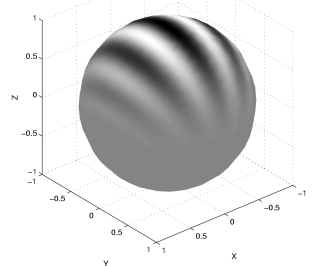

(a)

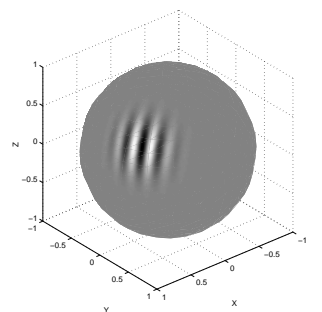

(c)

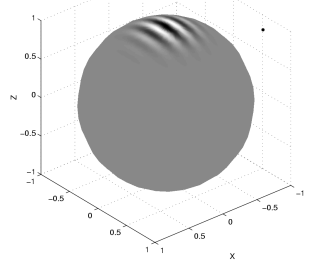

(b)

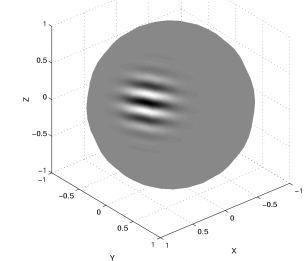

(d)

FIGURE 5 The spherical Morlet wavelet is shown at two scales, (a) $a=0.3$ and (b) $a=0.03$. Then displaced: (c) $a=0.03$, centered at $(\pi / 3, \pi / 3)$; and (d) The same, rotated by $\pi / 2$.

with inverse

$$
\left[\Pi^{-1} f\right](\theta, \varphi)=\frac{2}{1+\cos \theta} f(\mathrm{p}(\theta, \varphi)), \quad f \in L^{2}\left(\mathbb{R}^{2}, d^{2} \vec{x}\right) .
$$

Then every admissible Euclidean wavelet $\psi \in L^{2}\left(\mathbb{R}^{2}, d^{2} \vec{x}\right)$ yields an admissible spherical wavelet $\Pi^{-1} \psi \in L^{2}\left(S^{2}, d \mu\right)$. In particular, if $\psi$ is a directional wavelet, so is $\Pi^{-1} \psi$.

As an example, the (real part) of the spherical Morlet wavelet is shown in various positions in Figure 5. In order to show its directional selectivity, we present in Figure 6 the analysis of the triangle from Figure 3. The wavelet is oriented in two ways, $\chi=0^{\circ}$ and $\chi=90^{\circ}(\chi$ is the third Euler angle (see Section 2.1), which describes a rotation of the wavelet around its center). As expected, this wavelet filters out the directions perpendicular to its orientation, keeping the great $\operatorname{circles} \varphi=$ const. in the first case and the longitude $\operatorname{circles} \theta=$ const. in the second case.

\subsection{Wavelet frames on the 2-sphere}

In order to discretize our spherical CWT, we have to generalize the notion of frame. The classical notion [4] is that a countable family of vectors $\left\{\phi_{n}\right.$ : 


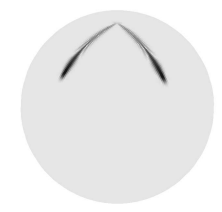

(a)

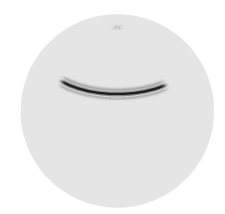

(b)

FIGURE 6 Analysis of a triangle with the spherical Morlet wavelet, in two different orientations: (a) $\chi=0^{\circ}$; (b) $\chi=90^{\circ}$, showing the directional selectivity of the wavelet.

$n \in \Gamma\}$ in a (separable) Hilbert space $\mathfrak{H}$ is a (discrete) frame if there exist two positive constants $\mathrm{m}>0$ and $\mathrm{M}<\infty$ such that

$$
\mathrm{m}\|f\|^{2} \leqslant \sum_{n \in \Gamma}\left|\left\langle\phi_{n} \mid f\right\rangle\right|^{2} \leqslant \mathrm{M}\|f\|^{2}, \text { for all } f \in \mathfrak{H} .
$$

The index set $\Gamma$ may be finite or infinite. We introduce two variants to this classical notion. The family $\left\{\phi_{n}\right\}$ is a controlled frame in $\mathfrak{H}$ if there is a positive bounded operator $C$, with bounded inverse, such that

$$
\mathrm{m}\|f\|^{2} \leqslant \sum_{n \in \Gamma}\left\langle\psi_{n} \mid f\right\rangle\left\langle f \mid C \phi_{n}\right\rangle \leqslant \mathrm{M}\|f\|^{2}, \text { for all } f \in \mathfrak{H} .
$$

The family $\left\{\phi_{n}\right\}$ is a weighted frame in $\mathfrak{H}$ if there are positive weights $w_{n}>0$ such that

$$
\mathrm{m}\|f\|^{2} \leqslant \sum_{n \in \Gamma} w_{n}\left|\left\langle\phi_{n} \mid f\right\rangle\right|^{2} \leqslant \mathrm{M}\|f\|^{2}, \text { for all } f \in \mathfrak{H} .
$$

These two notions are in fact mathematically equivalent to the classical notion of frame [6], namely, a family of vectors $\left\{\phi_{n}\right\}$ is a controlled frame, resp. a weighted frame, if and only if it is a frame in the standard sense (with different frame bounds, of course). However, this is not true numerically, the convergence properties of the respective frame expansions may be quite different $[4,6]$. And, indeed, the new notions are used precisely for improving the convergence of the reconstruction process.

\subsubsection{Half-continuous spherical frames}

In a first step, we will build a half-continuous spherical frame, by discretizing the scale variable only, while keeping continuous the position variable on the sphere (this is exactly the approach adopted by Duval-Destin et al. for designing the so-called continuous wavelet packets [14]).

Let us choose the half-continuous grid $\Lambda=\left\{\left(\omega, a_{j}\right): \omega \in S^{2}, j \in\right.$ $\left.\mathbb{Z}, a_{j}>a_{j+1}\right\}$, where $\mathcal{A}=\left\{a_{j}: j \in \mathbb{Z}\right\}$ is an arbitrary decreasing sequence of scales, and weights $\nu_{j}$ that mimic the natural (Haar) measure $d a / a^{3}$. 
If we start from the standard weighted frame condition given in (2.22), we do obtain a weighted frame, but there is no way of getting a tight one. The reason is obvious, the resolution operator $A_{\psi}$ has not been taken into account. We start instead from the Plancherel formula (2.16) and write a modified frame condition

$$
\mathrm{m}\|f\|^{2} \leqslant \sum_{j \in \mathbb{Z}} \nu_{j} \int_{S^{2}} d \mu(\omega) W_{f}\left(\omega, a_{j}\right) \overline{\widetilde{W}_{f}\left(\omega, a_{j}\right)} \leqslant \mathrm{M}\|f\|^{2} .
$$

To give an example, choose the axisymmetric SDOG wavelet $(2.13) \psi_{G}^{(\alpha)}(\alpha=$ 1.25). Then, proceeding as before, with the same weights $\nu_{j}$, one obtains that the ratio $\mathrm{M} / \mathrm{m}$ tends to 1 as the number of voices increases. Thus a tight frame might be obtained by this method. Indeed:

Proposition 2.5 Let $\mathcal{A}=\left\{a_{j}: j \in \mathbb{Z}\right\}$ be a decreasing sequence of scales. If $\psi$ is an axisymmetric wavelet for which there exist two constants $\mathrm{m}, \mathrm{M} \in \mathbb{R}_{+}^{*}$ such that

$$
\mathrm{m} \leqslant g_{\psi}(l) \leqslant \mathrm{M}, \text { for all } l \in \mathbb{N}
$$

where

$$
g_{\psi}(l)=\frac{4 \pi}{2 l+1} \sum_{j \in \mathbb{Z}} \nu_{j}\left|\widehat{\psi}_{a_{j}}(l, 0)\right|^{2},
$$

then any function $f \in L^{2}\left(S^{2}, d \mu\right)$ may be reconstructed from the corresponding family of spherical wavelets, as

$$
f(\omega)=\sum_{j \in \mathbb{Z}} \nu_{j} \int_{S^{2}} d \mu\left(\omega^{\prime}\right) W_{f}\left(\omega^{\prime}, a_{j}\right)\left[\ell_{\psi}^{-1} R_{\left[\omega^{\prime}\right]} D_{a_{j}} \psi\right]\left(\omega^{\prime}\right),
$$

where $\ell_{\psi}$ is the (discretized) resolution operator defined by $\widehat{\ell_{\psi}^{-1}} h(l, m)=$ $g_{\psi}(l)^{-1} h(l, m)$.

Note that the resolution operator $\ell_{\psi}$ is simply the discretized version of the continuous resolution operator $A_{\psi}$. Clearly (2.25) may be interpreted as a (weighted) tight frame controlled by the operator $\ell_{\psi}^{-1}$.

\subsubsection{Discrete spherical frames}

Next, we design a fully discrete spherical frame, by discretizing all the variables. The scale variable is discretized as before: $a \in \mathcal{A}=\left\{a_{j} \in \mathbb{R}_{+}^{*}: a_{j}>\right.$ $\left.a_{j+1}, j \in \mathbb{Z}\right\}$. As for the positions, we choose an equiangular grid $\mathcal{G}_{j}$ indexed by the scale level:

$$
\mathcal{G}_{j}=\left\{\omega_{j p q}=\left(\theta_{j p}, \varphi_{j q}\right) \in S^{2}: \theta_{j p}=\frac{(2 p+1) \pi}{4 B_{j}}, \varphi_{j q}=\frac{q \pi}{B_{j}}\right\},
$$

for $p, q \in \mathcal{N}_{j}:=\left\{n \in \mathbb{N}: n<2 B_{j}\right\}$ and some range of bandwidths $\mathcal{B}=$ $\left\{B_{j} \in 2 \mathbb{N}: j \in \mathbb{Z}\right\}$. 
Note that, in (2.26), the values $\left\{\theta_{j p}\right\}$ constitute a pseudo-spectral grid, with nodes on the zeros of a Chebyshev polynomial of degree $2 B_{j}$. Their virtue is the existence of an exact quadrature rule [13], namely,

$$
\int_{S_{2}} d \mu(\omega) f(\omega)=\sum_{p, q \in \mathcal{N}_{j}} w_{j p} f\left(\omega_{j p q}\right)
$$

for certain (explicit) weights $w_{j p}>0$ and for every band-limited function $f \in L^{2}\left(S^{2}, d \mu\right)$ of bandwidth $B_{j}$ (i.e., $\widehat{f}(l, m)=0$ for all $\left.l \geqslant B_{j}\right)$. Thus the complete discretization grid is $\Lambda(\mathcal{A}, \mathcal{B})=\left\{\left(a_{j}, \omega_{j p q}\right): j \in \mathbb{Z}, p, q \in \mathcal{N}_{j}\right\}$.

As before, we are looking for a controlled frame. The result [9] is that one can indeed get a discrete weighted, nontight, frame controlled by the operator $A_{\psi}^{-1}$, namely, $\left\{\psi_{j p q}=R_{\left[\omega_{j p q}\right]} D_{a_{j}} \psi: j \in \mathbb{Z}, p, q \in \mathcal{N}_{j}\right\}$ :

$$
\mathrm{m}\|f\|^{2} \leqslant \sum_{j \in \mathbb{Z}} \sum_{p, q \in \mathcal{N}_{j}} \nu_{j} w_{j p} W_{f}\left(\omega_{j p q}, a_{j}\right) \overline{\widetilde{W}}_{f}\left(\omega_{j p q}, a_{j}\right) \leqslant \mathrm{M}\|f\|^{2}
$$

A sufficient condition for (2.28) may be given, but it is very complicated, involving the determinant of an $\infty$-dimensional matrix, unless $f$ is bandlimited. Here again, the direct approach using a one voice discretization of the scale variable fails to yield a tight frame.

As usual, when the frame bounds are close enough, approximate reconstruction formulas may be used. The convergence of the process may still be improved by combining the reconstruction with a conjugate gradient algorithm. A spectacular example may be found in [9]. The signal is a World map, reconstructed with a half-continuous spherical frame and the SDOG wavelet, with a relative error of $1.1 \%$. Adding the conjugate gradient algorithm with 3 iterations only, the relative error drops to $2.10^{-3} \%$.

\subsection{Applications of the spherical CWT}

Up to now, the spherical CWT has been applied essentially in cosmology, notably the analysis of the fluctuations of the Cosmic Background radiation (CMB). Several astrophysics groups are by now using this approach, e.g. in Santander, Cambridge, EPFL, or in China (see for instance [22]). Two reviews of these applications, with references to previous work, may be found in this special volume, namely [37] and [24].

In addition, we present here another application, which exploits explicitly our spherical frames, namely, a local enhancement of Jupiter's Red Spot. The method runs as follows. Before reconstruction, the coefficients at the finest scale $W_{f}\left(\omega, a_{7}\right)$ are multiplied by a Gaussian mask

$$
M(\omega)=1+n_{a^{\prime}}\left(R_{\left[\omega^{\prime}\right]} D_{a^{\prime}} G\right)(\omega),
$$




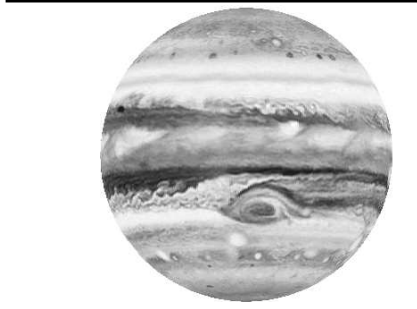

(a)

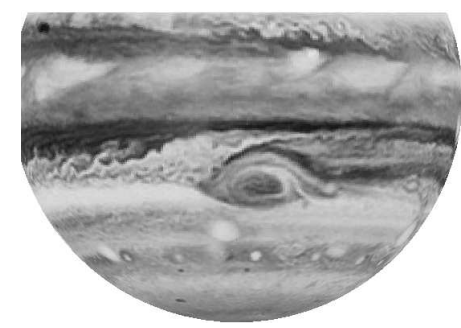

(c)

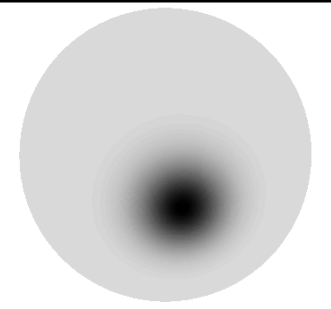

(b)

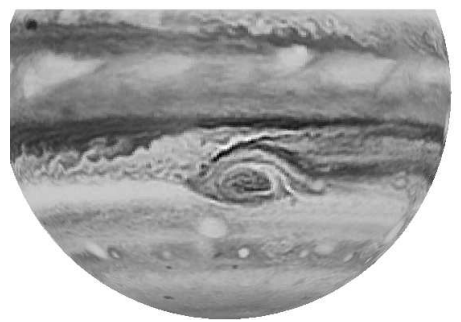

(d)

FIGURE 7 Local enhancement of Jupiter's Red Spot. (a) Original image; (b) Local mask; (c) Zoom over the Red Spot; (d) Zoom over the Red Spot with sharper details.

localized on the center $\omega^{\prime}$ of the Spot. Here $G$ is a Gaussian (this is only for convenience), $a^{\prime}$ a dilation factor such that the dilated Gaussian covers the entire Spot and $n_{a^{\prime}}$ a normalization factor ensuring that $\|M\|_{\infty}=2$. This mask increases the amplitudes of the fine scale coefficients by a factor up to 2 in the vicinity of the Red Spot, but the rest of the coefficients are not modified (the mask is thus a frame multiplier [6]). The reconstruction is made with a half-continuous spherical frame with a SDOG wavelet and an equiangular grid of size $512 \times 512$, which gives a good discretization for $|j| \leqslant$ 7. The tools are the SpharmonicKit package [28] and our own MATLAB ${ }^{\circledR}$ YAWtb toolbox [38]. The result is shown in Figure 7. Clearly, such a technique is impossible to implement with a purely frequential spherical decomposition; one really needs a spherical wavelet frame.

\subsection{Recent developments}

New techniques for spherical wavelets continue to emerge, mostly motivated by the astrophysical applications described above. For instance, an alternative approach to the spherical CWT has been proposed very recently [23, 31]. The idea is to avoid the stereographic projection and to define dilations by a scaling operation in spherical harmonic space (that is, effectively, in Fourier space), an idea that goes back to Holschneider [19] and Freeden [15]. These 
new wavelets have been applied successfully to the detection of point sources, but the full potential of the method has not yet been analyzed.

On the other hand, a new class of discrete spherical wavelets, called needlets, has been introduced [7, 25]. These functions are derived by combining three ideas, namely, a Littlewood-Paley decomposition, a suitable distribution of (finitely many) points on the sphere, called centers, and an exact quadrature rule. The upshot is a new class of tight frames on the sphere. The frame functions, which are actually special spherical harmonics kernels, are both compactly supported in the frequency domain (i.e., bandlimited in $l$ ) and almost exponentially localized around each center. When combined with a new statistical method, they offer a powerful tool for analyzing CMB (WMAP) data, in particular analyzing the cross-correlation between the latter and galaxy counts from sky surveys [26].

\section{Outcome: The CWT on other conic sections}

According to Apollonius, the conic sections are the sphere $S^{2}$, the paraboloid $P^{2}$ and the two-sheeted hyperboloid $H^{2}$. All three are obtained as sections by a plane of a double null-cone

$$
\mathcal{C}_{0}^{3}=\left\{\left(x_{0}, x_{1}, x_{2}, x_{3}\right) \in \mathbb{R}^{4}: x_{0}^{2}-x_{1}^{2}-x_{2}^{2}-x_{3}^{2}=0\right\} .
$$

As we have seen in the previous section, there is a completely satisfactory CWT on the two-sphere. What about the other cases? Interesting results have been obtained, but much work still lies ahead. We will report in detail on these nonspherical cases in a further publication [5] and give here only a glimpse.

\section{(1) The two-sheeted hyperboloid}

$H^{2}$ is the dual manifold of the sphere $S^{2}$, with constant negative curvature and a large isometry group, namely $\mathrm{SO}_{o}(2,1)$. As in the case of the sphere, the latter yields motions on the manifold, but here dilations are problematic. The hyperbolic analogue of the stereographic dilation is inadequate, since large stereographic dilations map the upper sheet of $H^{2}$ onto the lower sheet. Several other methods are available, however. In addition, a reconstruction formula may be derived, with help of an appropriate integral transform, the Fourier-Helgason transform, that defines harmonic analysis on $H^{2}$, including convolution theorems. Thus there exists a satisfactory CWT on $H^{2}$, even several, but no results are known concerning frames.

\section{(2) The paraboloid}

This is a limit case between the sphere $S^{2}$ and the two-sheeted hyperboloid $H^{2}$. Actually the paraboloid $P^{2}$ does not have a constant curvature, and there is no large isometry group, so that the general method does not 
work. Therefore, designing a CWT on $P^{2}$, by a limiting procedure or otherwise, is bound to be a hard process. One can design a certain integral transform, but it does not involve proper dilations, hence it is not really a wavelet transform.

\section{(3) A unified approach to all conic sections}

All conic sections may be obtained simply by varying the tilt angle $\alpha$ of the plane intersecting the null-cone (3.1), i.e., writing the equation of the plane as $x_{0}=1+\tan \alpha\left(x_{3}-2\right), 0 \leqslant \alpha \leqslant \pi$. Thus it is tempting to try to design a unified CWT. Several methods may be used, mostly relying on group theory. The approach looks promising, but still incomplete.

The conclusion of this review is that wavelets on non-Euclidean manifolds are an active field of research, that keeps finding new and exciting applications, mostly, but not only, in astrophysics. Progress is also made on the mathematical side, but much work remains to be done, especially on the nonspherical cases.

\section{Appendix: Some key facts from group theory}

We collect here some key facts from group theory which are used in the text. Further information may be found in standard textbooks, such as [8] or [17].

All the groups mentioned in the text are matrix groups, namely the orthogonal groups $\mathrm{SO}(2), \mathrm{SO}(3)$ and the pseudo-orthogonal ones $\mathrm{SO}_{o}(1,3)$, $\mathrm{SO}_{o}(1,2)$. These are in fact Lie groups, that is, groups $G$ which are at the same time smooth manifolds such that the map $G \times G \rightarrow G,(g, h) \mapsto g h^{-1}$ is (infinitely often) differentiable.

If $G$ is a Lie group and $H$ a closed subgroup of $G$, then the quotient $G / H$, that is, the set of left cosets $g H$, is a smooth manifold. For instance, $\mathrm{SO}(3) / \mathrm{SO}(2) \simeq S^{2}$, the two-sphere, and the two are isomorphic smooth manifolds. Given a quotient $X=G / H$, a section is a map $\sigma: X \rightarrow G$ such that the composed map $\pi \circ \sigma: X \rightarrow X$ is the identity; here $\pi: G \rightarrow X$ is the (canonical) map $g \mapsto g H$.

An isomorphism between two groups $G, G^{\prime}$ is a bijection $\beta: G \rightarrow G^{\prime}$ that preserves the group law: $\beta\left(g_{1} g_{2}\right)=\beta\left(g_{1}\right) \beta\left(g_{2}\right)$, for all $g_{1}, g_{2} \in G$. An automorphism of $G$ is an isomorphism $\beta: G \rightarrow G$. An inner automorphism is simply the conjugation by an element $h \in G: g \mapsto h g h^{-1}$, for all $g \in G$. An outer automorphism of $G$ is an automorphism which is not inner.

Let $H, K$ be two groups for which there exists a map (in fact, a homomorphism) $\alpha$ from $K$ to the automorphisms of $H$. Then the semidirect product of $H$ (noted additively) by $K$ (noted multiplicatively), with respect to the map $\alpha$, is the group $G=H \rtimes K$ of all pairs $(h, k) \in H \times K$, with composition law

$$
(h, k)\left(h^{\prime}, k^{\prime}\right)=\left(h+\alpha(k)\left(h^{\prime}\right), k k^{\prime}\right), \text { for all }(h, k),\left(h^{\prime}, k^{\prime}\right) \in H \times K .
$$


A typical example is the Euclidean group $\mathrm{E}(3)=\mathbb{R}^{3} \rtimes \mathrm{SO}(3)$, with the composition law $(x, R)\left(x^{\prime}, R^{\prime}\right)=\left(x+R x^{\prime}, R R^{\prime}\right)$, for all $(x, R),\left(x^{\prime}, R^{\prime}\right) \in$ $\mathbb{R}^{3} \times \mathrm{SO}(3)$. Thus, if the group $K$ has no (outer) automorphism (i.e., $\alpha(k) \equiv$ identity), the only possibility for building a larger group out of $H$ and $K$ is to take their direct product - but then the two factors must commute: $h k \equiv\left(h, e_{K}\right)\left(e_{H}, k\right)=(h, k)=\left(e_{H}, k\right)\left(h, e_{K}\right) \equiv k h\left(e_{H}, e_{K}\right.$ are the units).

The Iwasawa decomposition: every connected Lie group $G$ may be decomposed uniquely into a product of three subgroups, $G=K A N$, where $K$ is the maximal compact subgroup, $A$ is abelian and $N$ is nilpotent $[8,18]$.

If $G$ is a group, with unit $e$, and $X$ is a set, an action of $G$ on $X$ is a map $(g, x) \in G \times X \mapsto g[x] \in X$ such that (i) $g_{1} g_{2}[x]=g_{1}\left[g_{2}[x]\right]$, for all $g_{1}, g_{2} \in G$, for all $x \in X$, and (ii) $e[x]=x, e \in G$, for all $x \in X$. The action of $G$ on $X$ is transitive if, for every pair $x, x^{\prime} \in X$, there exists an element $g \in G$ such that $x=g\left[x^{\prime}\right]$. The canonical example is the action of a group $G$ on a quotient $G / H, g\left[g^{\prime} H\right]=\left(g g^{\prime}\right) H$, which is then called a homogeneous space. For instance, the Lorentz group $\mathrm{SO}_{o}(1,3)$ acts transitively on the two-sphere $S^{2}$.

Every locally compact group, in particular, every Lie group, possesses a unique (up to normalization) left invariant measure $\mu$ (called a Haar measure), that is, a measure $\mu$ on $G$ which satisfies the following relation for any $\mu$-integrable function $f$ :

$$
\int_{G} f\left(g_{0} g\right) d \mu(g)=\int_{G} f(g) d \mu(g), \text { for all } g_{0} \in G,
$$

or, equivalently,

$$
d \mu\left(g_{0}^{-1} g\right)=d \mu(g) .
$$

A unique right invariant measure also exists and it is equivalent to the left invariant one. The Haar measures allow a full theory of integration on a locally compact group.

A unitary representation of a group $G$ in a Hilbert space $\mathfrak{H}$ is a homomorphism $U$ of $G$ into the unitary operators on $\mathfrak{H}$ :

$$
U\left(g_{1} g_{2}\right)=U\left(g_{1}\right) U\left(g_{2}\right), \text { for all } g_{1}, g_{2} \in G .
$$

It follows that $U\left(g^{-1}\right)=U(g)^{-1}=U(g)^{*}$ and $U(e)=I$. The representation is irreducible if the only closed subspaces of $\mathfrak{H}$ which are invariant under $U$ are the trivial subspace $\{0\}$ and $\mathfrak{H}$ itself. The unitary irreducible representation $U$ is square integrable if it possesses a nonzero (admissible) vector $\eta \in \mathfrak{H}$ such that

$$
\int_{G}|\langle U(g) \eta \mid \eta\rangle|^{2} d \mu(g)<\infty
$$

where $\mu$ is the left Haar measure on $G$, or, equivalently,

$$
\int_{G}|\langle U(g) \eta \mid \phi\rangle|^{2} d \mu(g)<\infty, \text { for all } \phi \in \mathfrak{H} .
$$


The notion of square integrability of a representation may be extended to a quotient, as follows (compare (2.7)). Let $X=G / H$ be a homogeneous space of $G$, with left invariant measure $\nu$, and $\sigma: X \rightarrow G$ a suitable section. Then the unitary irreducible representation $U$ is square integrable on $X$ for the section $\sigma$ if there exist a nonzero (admissible) vector $\eta \in \mathfrak{H}$ such that

$$
\int_{X}|\langle U(\sigma(g)) \eta \mid \phi\rangle|^{2} d \nu(x)<\infty, \text { for all } \phi \in \mathfrak{H} \quad(x \equiv g H) .
$$

[1] Antoine, J-P. and Vandergheynst, P. (1998). Wavelets on the $n$-sphere and other manifolds, J. Math. Phys. 39, 3987-4008.

[2] Antoine, J-P. and Vandergheynst, P. (1999). Wavelets on the 2-sphere: A grouptheoretical approach, Applied Comput. Harmon. Anal. 7, 262-291.

[3] Antoine, J-P., Demanet L., Jacques, L., and Vandergheynst, P. (2002). Wavelets on the sphere: Implementation and approximations, Applied Comput. Harmon. Anal. 13, 177-200.

[4] Antoine, J-P., Murenzi, R., Vandergheynst, P., and Ali, S.T. (2004). TwoDimensional Wavelets and their Relatives, Cambridge Univ. Press, Cambridge, UK.

[5] Antoine, J-P., Bogdanova, I., and Vandergheynst, P. (2007). The continuous wavelet transform on conic sections (in preparation).

[6] Balazs, P. and Antoine, J-P. (2006). Weighted and controlled frames, preprint UCLIPT-06-05.

[7] Baldi, P., Kerkyacharian, G, Marinucci, D., and Picard, D. (2006). Asymptotics for spherical needlets. arXiv:math.ST/0606599 v1, Sep. 2006.

[8] A. O. Barut and R. Rączka (1977). Theory of Group Representations and Applications. PWN, Warszawa.

[9] Bogdanova, I., Vandergheynst, P., Antoine, J-P., Jacques, L., and Morvidone, M. (2005). Stereographic wavelet frames on the sphere, Applied Comput. Harmon. Anal. 26, 223-252.

[10] Bülow, T. (2002). Multiscale image processing on the sphere, in DAGM-Symposium, pp. 609-617.

[11] Dooley, A.H. and Rice, J.W. (1983) Contractions of rotation groups and their representations, Math. Proc. Camb. Phil. Soc. 94, 509-517.

[12] Dooley, A.H. and Rice, J.W. (1985). On contractions of semisimple Lie groups, Trans. Amer. Math. Soc. 289, 185-202.

[13] Driscoll, J.R. and Healy, D.M. (1994). Computing Fourier transforms and convolutions on the 2-sphere, Adv. Appl. Math. 15, 202-250.

[14] Duval-Destin, M. Muschietti, M-A., and Torrésani, B. (1993). Continuous wavelet decompositions, multiresolution, and contrast analysis, SIAM J. Math. Anal. 24, 739-755.

[15] Freeden, W. and Windheuser, U. (1997). Combined spherical harmonic and wavelet expansion - A future concept in Earth's gravitational determination, Applied Comput. Harmon. Anal. 4, 1-37.

[16] Freeden, W., Maier, T., and Zimmermann, S. (2003). A survey on wavelet methods for (geo)applications, Revista Mathematica Complutense 16, 277-310.

[17] R. Gilmore (1974). Lie Groups, Lie Algebras, and Some of Their Applications. Wiley, New York and London.

[18] S. Helgason (1962). Differential Geometry and Symmetric Spaces. Academic Press, New York and London.

[19] Holschneider, M. (1996). Continuous wavelet transforms on the sphere, J. Math. Phys. 37, 4156-4165. 
[20] Jacques, L. (2004). Ondelettes, repères et couronne solaire. Thèse de Doctorat, Univ. Cath. Louvain, Louvain-la-Neuve.

[21] Kunoth, A. and Sahner, J. (2006). Wavelets on manifolds: An optimized construction, Math. Comput. 75 (255), 1319-1349.

[22] Liu X. and Zhang S.N. (2005). Non-Gaussianity due to possible residual foreground signals in Wilkinson Microwave Anistropy Probe first-year data using spherical wavelet approaches, Astrophys. J. 633, 542-551.

[23] McEwen, J.D., Hobson, M.P., and Lasenby, A.N. (2006). A directional continuous wavelet transform on the sphere, arXiv:astro-ph/0609159 v1, Sep. 2006.

[24] McEwen, J.D., Vielva, P., Wiaux, Y., Barreiro, R.B., Cayon, L., Hobson, M.P., Lasenby, A.N., and Martinez-Gonzalez, E. (2006). Cosmological applications of a wavelet analysis on the sphere, J. Fourier Anal. Appl. 13, this volume.

[25] Narcowich, F. J., Petrushev, P., and Ward, J. D. (2006). Localized tight frames on spheres, SIAM J. Math. Anal. 38, 574-594.

[26] Pietrobon, D., Balbi, A., and Marinucci, D. (2006). Integrated Sachs-Wolfe effect from the cross correlation of WMAP3 year and the NRAO VLA sky survey data: New results and constraints on dark energy arXiv:astro-ph/0606475 v2, Sep. 2006.

[27] Potts, D., Steidl, G., and Tasche, M. (1996). Kernels of spherical harmonics and spherical frames, in Advanced Topics in Multivariate Approximation, F. Fontanella, K. Jetter and P.J. Laurent (eds.), World Scientific, Singapore, pp. 287-301.

[28] Rockmore, D., Moore, S., Healy, D., and Kostelec, P. SpharmonicKit (Dartmouth College) http://www.cs.dartmouth.edu/ geelong/sphere/.

[29] Roşca, D. (2005). Locally supported rational spline wavelets on the sphere, Math. Comput. 74 (252), 1803-1829.

[30] Roşca, D. (2007). Wavelet bases on the sphere obtained by radial projection, $J$. Fourier Anal. Appl. 13, this volume.

[31] Sanz, J.L., Herranz, D., López-Caniego, M., and Argüeso, F. (2006). Wavelets on the sphere. Application to the detection problem, arXiv:astro-ph/0609351 v1, Sep. 2006 .

[32] Schröder, P. and Sweldens, W. (1995). Spherical wavelets: Efficiently representing functions on the sphere, in Computer Graphics Proceedings (SIGGRAPH1995), SIGGRAPH, ACM, pp. 161-172.

[33] Takahashi, R. (1963). Sur les représentations unitaires des groupes de Lorentz généralisés, Bull. Soc. Math. France 91, 289-433.

[34] Torrésani, B. (2005). Position-frequency analysis for signals defined on spheres, Signal Proc. 43, 341-346.

[35] Weinreich, I. (2001). A construction of $C^{1}$-wavelets on the two-dimensional sphere, Applied Comput. Harmon. Anal. 10, 1-26.

[36] Wiaux, Y., Jacques, L., and Vandergheynst P. (2005). Correspondence principle between spherical and Euclidean wavelets, Astrophys. J. 632, 15-28.

[37] Wiaux, Y., McEwen, J.D., and Vielva P. (2007). Complex data processing: Fast wavelet analysis on the sphere, J. Fourier Anal. Appl. 13, this volume.

[38] http://www.fyma.ucl.ac.be/projects/yawtb.

$$
\text { Received ...., } 2007
$$

Institut de Physique Théorique, Université catholique de Louvain

$$
\text { B - } 1348 \text { Louvain-la-Neuve, Belgium }
$$

antoine@fyma.ucl.ac.be

Signal Processing Institute, Swiss Federal Institute of Technology, EPFL

Station 11, CH-1015 Lausanne, Switzerland

pierre.vandergheynst@epfl.ch 